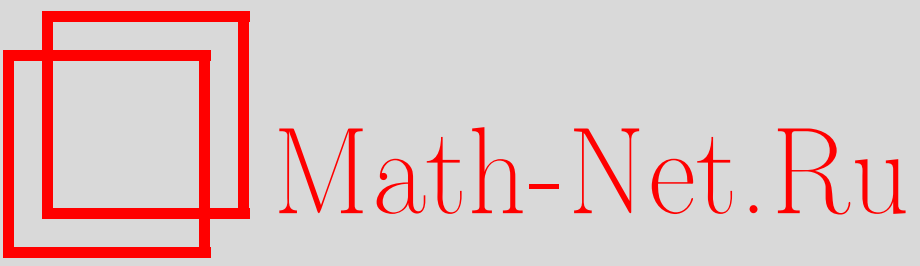

Д. В. Чистяков, Фрактальная геометрия образов непрерывных вложений $p$ адических чисел и соленоидов в евклидовы пространства, ТМФ, 1996, том 109, номер 3, 323-337

DOI: https://doi.org/10.4213/tmf1230

Использование Общероссийского математического портала Math-Net.Ru подразумевает, что вы прочитали и согласны с пользовательским соглашением

http: //www . mathnet.ru/rus/agreement

Параметры загрузки:

IP : 54.224 .187 .69

26 апреля 2023 г., 11:05:01

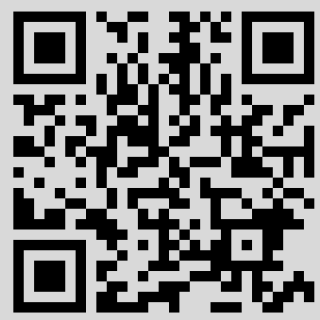




\title{
Д. В. Чистяков* \\ ФРАКТАЛЬНАЯ ГЕОМЕТРИЯ ОБРАЗОВ НЕПРЕРЫВНЫХ ВЛОЖЕНИЙ $p$-АДИЧЕСКИХ ЧИСЕЛ И СОЛЕНОИДОВ В ЕВКЛИДОВЫ ПРОСТРАНСТВА
}

\begin{abstract}
Получены явные формулы для семейства непрерывных отображений $p$-адических чисел $\mathbf{Q}_{p}$ и соленоидов $\mathbf{T}_{p}$ в комплексную плоскость $\mathbf{C}$ и $\mathbf{R}^{3}$, соответственно. Такому семейству принадлежат отображения, для которых множество Кантора и ковер Серпинского суть образы единичного шара в $\mathbf{Q}_{2}$ и $\mathbf{Q}_{3}$, соответственно. В каждом семействе найдено подмножество вложений. Для этих вложений вычислены хаусдорфовы размерности и показано, что фрактальная мера на образе $\mathbf{Q}_{p}$ совпадает с мерой Хаара в $\mathbf{Q}_{p}$. Показано, что при определенных условиях образ $p$-адического соленоида есть инвариантное множество дробной размерности некоторой динамической системы. Приведены компьютерные рисунки некоторых фрактальных образов.
\end{abstract}

Иерархическая структура $p$-адических чисел и фракталов, а также симметрии самоподобных фракталов указывают на тесную связь между этими объектами, что неоднократно подмечалось [1-3]. Яркий пример - гомеоморфность канторовского множества и кольца целых 2 -адических чисел $\mathbf{Z}_{2}[1,2,4]$. Между тем, насколько известно автору, изучению явных формул вложений различных подмножеств $\mathbf{Q}_{p}$ в евклидовы пространства и изучению фрактальных свойств соответствующих образов не было уделено достаточного внимания. Вместе с тем такие объекты как странные аттракторы [5] имеют топологию, сходную с топологией $p$-адических соленоидов $\mathbf{T}_{p}$, поэтому построение вложений $\mathbf{T}_{p}$, скажем, в трехмерное евклидово пространство $\mathbf{R}^{3}$ также может представлять интерес.

В данной работе построены непрерывные отображения $\Upsilon_{s}^{(m)}: \mathbf{Q}_{p} \mapsto \mathbf{C}$ и $\Omega_{s, a}^{(m)}: \mathbf{T}_{p} \mapsto$ $\mathbf{R}^{3}$, зависяшие от параметров $s, a \in \mathbf{C}$ и натурального или равного $\infty$ числа $m$. Оказалось, что $\Upsilon_{1 / 3}^{(0)}\left(\mathbf{Z}_{2}\right)$ есть множество Кантора и $\Upsilon_{1 / 2}^{(0)}\left(\mathbf{Z}_{3}\right)$ - ковер Серпинского. Показано, что для некоторого $s_{0}>0$ условие $|s|<s_{0}$ (и $\left.|a|<(1-|s|)^{-1}\right)$ определяет множества отображений $\Upsilon_{s}^{(m)}$ и $\Omega_{s, a}^{(m)}$, которые являются вложениями. Эти множества вложений обладают таким свойством: для указанных $s$ и $a$ можно подобрать аддитивно инвариантные метрики в $\mathbf{Q}_{p}$ и $\mathbf{T}_{p}$ так, что отображения $\Upsilon_{s}^{(m)}$ и $\Omega_{s, a}^{(m)}$ будут сохранять хаусдорфовы размерности любых подмножеств. Это свойство оказывается удобным при изучении хаусдорфовых мер множеств $\Upsilon_{s}^{(m)}\left(\mathbf{Q}_{p}\right)$ и $\Omega_{s, a}^{(m)}\left(\mathbf{T}_{p}\right)$, поскольку хаусдорфовы меры в $\mathbf{Q}_{p}$ и $\mathbf{T}_{p}$ с соответствуюшими метриками суть просто меры Хаара. Более того, оказывается, что мера Хаара любого множества в $\mathbf{Q}_{p}$ совпадает с фррактальной мерой его

* Казанский государственный университет 
образа (см. формулу (22)). Это обстоятельство, а также тот факт, что $\Upsilon_{s}^{(\infty)}$ представляет собой ряд из непрерывных аддитивных характеров на $\mathbf{Q}_{p}$, позволяют использовать теоретико-групповые методы при вычислении интегралов по фрактальной мере. Это может оказаться эффективным средством решения некоторых задач квантовой механики, диффузии или дифракции на таких фракталах $[3,6,7]$.

Удалось доказать, что для вложений $\Omega_{s, a}^{(\infty)}$ существует динамическая система ${ }^{1)}$ такая, что $\Omega_{s, a}^{(m)}\left(\mathbf{T}_{p}\right)$ есть инвариантное множество этой системы, и любая интегральная траектория, лежашая в $\Omega_{s, a}^{(m)}\left(\mathbf{T}_{p}\right)$, плотно наматывается на это множество. Кроме того, отображения $\Upsilon_{s}^{(\infty)}$ и $\Omega_{s, a}^{(\infty)}$ связаны друг с другом. В данной работе построен непрерывный инъективный гомоморфизм $j$ адлитивной группы $\mathbf{Q}_{p}$ в группу $\mathbf{T}_{p}$ такой, что $j\left(\mathbf{Q}_{p}\right)$ плотен в $\mathbf{T}_{p}$, и доказано сушествование липшицева отображения $J: \mathbf{C} \mapsto \mathbf{R}^{3}$, коммутируюшего с $j$ (см. формулу (41)), такого, что сужение его на $\Upsilon_{s}^{(m)}\left(\mathbf{Q}_{p}\right)$ есть локальная изометрия.

\section{1. ХАУСДОРФОВЫ МЕРЫ (ОСНОВНЫЕ ОПРЕДЕЛЕНИЯ)}

Здесь мы приводим необходимые сведения о мерах Хаусдорфа в той форме, которая удобна для дальнейшего изложения. Для произвольного метрического пространства $(M, \rho)$ определим $\delta$-мерную внешнюю хаусдорфову меру $h^{\delta}$, положив $\forall A \subset M$

$$
h^{\delta}(A)=\lim _{\varepsilon \rightarrow+0} h_{\varepsilon}^{\delta}(A)=\lim _{\varepsilon \rightarrow+0} \inf \left\{\sum_{i=1} \operatorname{diam}\left(S_{i}\right)^{\delta}: \bigcup_{i=1}^{\infty} S_{i} \supseteq A, \operatorname{diam}\left(S_{i}\right) \leq \varepsilon\right\},
$$

где $\delta$ - фиксированное число большее 0 и $\operatorname{diam}(B) \equiv \sup \{\rho(x, y): x, y \in B\}$. Тогда $h^{\delta}$ есть счетно-аддитивная борелевски регулярная мера [8]. Хаусдорфова размерность $\forall A \subset M$ по определению есть число [9]

$$
D_{h}(A)=\inf \left\{\delta: h^{\delta}(A)=0\right\}=\sup \left\{\delta: h^{\delta}(A)=\infty\right\} .
$$

Из этого, в частности, следует, что если $h^{\delta}(A)>0$ и мера $h_{A}^{\delta}(\cdot) \equiv h^{\delta}(\cdot \cap A) \quad \sigma$-конечна, то $\delta=D_{h}(A)$. Под локальной хаусдорфовой размерностью в точке $x \in A$ будем понимать число $D_{h}^{L}(x)=\inf \left(D_{h}\left(A \cap U_{x}\right)\right)$, где inf берется по всем открытым окрестностям $U_{x}$ точки $x$.

СоглАшЕниЕ. Независимо от природы множества $X$ для любых действительных неотрицательных функций $F$ и $G$ на $X$ будем писать $F \stackrel{L}{\preceq} G$ или $\forall x \in X \quad F(x) \stackrel{L}{\preceq} G(x)$, как только найдется такое $C>0$, что $\forall x \in X \quad F(x) \leq C G(x)$, и если одновременно $F \stackrel{L}{\preceq} G$ и $G \stackrel{L}{\preceq} F$, то $F \stackrel{L}{\simeq} G$, и будем говорить, что функции $F$ и $G$ эквивалентны ${ }^{2}$. Понимая константы как постоянные функции, вместо $0<c<\infty$ будем писать $c \stackrel{L}{\simeq} 1$. Для любых таких $F, G$ и $H \stackrel{L}{\preceq} F$ выполнены следуюшие элементарные соотношения: $\forall a, b, \alpha>0$

$$
\begin{gathered}
\min (F, H) \stackrel{L}{\simeq} H, \quad \max (F, H) \stackrel{L}{\simeq} F \\
a F+b G \stackrel{L}{\simeq} \max (F, G) \stackrel{L}{\simeq} \max (F, G \pm H) \stackrel{L}{\simeq}\left(F^{\alpha}+G^{\alpha}\right)^{\frac{1}{\alpha}} .
\end{gathered}
$$

\footnotetext{
1) Под динамической системой в $\mathbf{R}^{n}$ мы понимаем автономную систему $n$ уравнений первого порядка, удовлетворяющую условиям теоремы существования и единственности.

${ }^{2)}$ На самом деле символы $\stackrel{L}{\preceq}, \underset{\sim}{\simeq}$ определяют отношения порядка и эквивалентности.
} 
Пусть дано отображение $\Phi: M \mapsto N$ метрических пространств $(M, \rho)$ и $(N, d)$ и пусть $d^{\Phi} \equiv d(\Phi(\cdot), \Phi(\cdot))$, тогда если $d^{\Phi} \stackrel{L}{\preceq} \rho$, то $\Phi$ - липшицево отображение. Назовем тогда $\Phi L$-сжсатием, и если $d^{\Phi} \stackrel{L}{\simeq} \rho$, то $\Phi$ - липшицево вложение и сужение $\Phi^{-1}$ на $\operatorname{Ran}(\Phi)$ также липшицево; такое отображение будем называть липииц-изометрией или L-изометрией.

ОПРЕДЕЛЕНИЕ 1 . Пусть на множестве $M$ заданы две псевдометрики $\rho_{1}$ и $\rho_{2}$, назовем расхождением $\rho_{1}$ и $\rho_{2}$ величину (полагая здесь, что $0 / 0=0$ )

$$
\kappa_{1,2}=\sup _{x, y \in M}\left(\frac{\left|\rho_{1}(x, y)-\rho_{2}(x, y)\right|}{\rho_{1}(x, y)+\rho_{2}(x, y)}\right) .
$$

Очевидно, что $\kappa_{1,2} \leq 1$, более того, можно показать, что $\kappa_{1,2}<1$ тогда и только тогда, когда $\rho_{1} \stackrel{L}{\simeq} \rho_{2}$. Нетрудно показать, что справедлива следуюшая

Теорема 1. Пусть $\rho_{1}, \rho_{2}$ - метрики на $M, \kappa_{1,2}-$ их расхождение и $h_{1}^{\delta}, D_{1}$, $D_{1}^{L}$ и $h_{2}^{\delta}, D_{2}, D_{2}^{L}$ обозначают соответствующие $\delta$-мерные мерь, глобальные и локальные размерности Хаусдорфа. Тогда справедливо соотношение

$$
\left(\frac{1-\kappa_{1,2}}{1+\kappa_{1,2}}\right)^{\delta} h_{1}^{\delta}(F) \leq h_{2}^{\delta}(F) \leq\left(\frac{1+\kappa_{1,2}}{1-\kappa_{1,2}}\right)^{\delta} h_{1}^{\delta}(F),
$$

из которого следует, что если $\rho_{1} \stackrel{L}{\simeq} \rho_{2}$, mо $h_{1}^{\delta} \stackrel{L}{\simeq} h_{2}^{\delta} u \forall F \subset M \quad D_{h, 1}(F)=D_{h, 2}(F)$ $u D_{h, 1}^{L}(x)=D_{h, 2}^{L}(x) \quad \forall x \in F$.

Из теорем 1 и 2.10.45 из [8] с учетом (3) вытекает

Tеорема 2. Пусть дано метрическое пространство $(M, \rho)$, в $\mathbf{R} \times M$ введем метрику d такую, что $d((x, a),(y, b))=\max (|x-y|, \rho(a, b)), \forall x, y \in \mathbf{R} u \forall a, b \in M$. Тогда для всякого борелевского $A \subset \mathbf{R} u \forall B \subset M$ такого, ито $h^{\delta}(B)<\infty$,

$$
h^{\delta+1}(A \times B) \stackrel{L}{\simeq} h^{1}(A) h^{\delta}(B)
$$

\section{2. МЕРЫ ХАУСДОРФА В $\mathbf{Q}_{p}$ И $\mathbf{T}_{p}$}

В этом разделе $\mathbf{Q}_{p}$ и $\mathbf{T}_{p}$ рассматриваются как пополнения $\mathbf{Q}$ по соответствующим аддитивно инвариантным метрикам.

Каждый элемент $x$ поля $p$-адических чисел $\mathbf{Q}_{p}$ однозначно представляется формальным рядом [10]:

$$
x=\sum_{n=v}^{\infty} a_{n} p^{n}=\sum_{n=v}^{-1} a_{n} p^{n}+\sum_{n=0}^{\infty} a_{n} p^{n},
$$

с коэффициентами $a_{n} \in\{0,1, \ldots, p-1\}, v<\infty$ для некоторого фиксированного простого $^{3)}$ числа $p$. Этот ряд абсолютно сходится по $p$-адической норме, которая $\forall x$ по определению равна $\|x\|=p^{-\alpha v(x)}$ для некоторого $\alpha>0, v(x)=v$ называют логарифмической

\footnotetext{
3) На самом деле можно выбрать $p$ любым натуральным числом, т.к. мы нигде не используем факт существования обратных элементов в кольце $\mathbf{Q}_{p}$.
} 
нормой $x$. Первая сумма в (6) обозначается как $\{x\}_{p}$ - дробная часть $x$, вторая - $[x]_{p}-$ целая часть $x$, при этом $\{x\}_{p} \in \mathbf{Q} \cap[0,1)$ и $[x]_{p} \in \mathbf{Z}_{p}$. Здесь $\mathbf{Z}_{p}=\left\{x \in \mathbf{Q}_{p}:|x|_{p} \leq 1\right\}-$ кольцо целых $p$-адических чисел. Любое число $q \in \mathbf{Q}$ однозначно разлагается в ряд (6), и $\mathbf{Q}_{p}$ есть пополнение $\mathbf{Q}[10]$. Норма $\|\cdot\|$ обладает свойством ультраметричности, а именно $\forall x, y \in \mathbf{Q}_{p}$

$$
\|x-y\| \leq \max (\|x\|,\|y\|) .
$$

Норму с $\alpha=1$ будем обозначать как $|\cdot|_{p}$, а остальные - просто как $|\cdot|_{p}^{\alpha}$. Все эти нормы, зависящие от $\alpha$, топологически эквивалентны [10], однако хаусдорфовы меры различны (см. ниже).

Стандартная мера Хаара $\chi$ в $\mathbf{Q}_{p}$ выбирается так, чтобы [10]

$$
\chi\left(\mathbf{Z}_{p}\right)=\int_{\mathbf{z}_{p}} d \chi=1 .
$$

Теорема 3. Хаусдорфова мера $h^{1 / \alpha}$ на $\left(\mathbf{Q}_{p},|\cdot|{ }_{p}^{\alpha}\right)$ есть стандартная борелевски регулярная мера Хаара $\forall \alpha>0, u$, следовательно, локальная и глобальная хаусдорфовь размерности $\left(\mathbf{Q}_{p},|\cdot|_{p}^{\alpha}\right)$ совпадают и равны $1 / \alpha$.

ДокАЗАТЕЛЬСтво. По построению $\forall \delta>0 \quad h^{\delta}$ - инвариантная мера, поэтому из единственности $\chi$ следует, что достаточно показать, например, что $h^{1 / \alpha}\left(\mathbf{Z}_{p}\right)=$ $\chi\left(\mathbf{Z}_{p}\right)=1$. Поскольку $\mathbf{Z}_{p}$ состоит ровно из $p^{M}$ непересекающихся шаров диаметра $p^{-M \alpha} \forall M \in \mathbf{N}$, то $h^{1 / \alpha}\left(\mathbf{Z}_{p}\right) \leq 1$. Покажем теперь, что $h^{1 / \alpha}\left(\mathbf{Z}_{p}\right) \geq 1$. Действительно, благодаря полуаддитивности $\chi \forall A \subset \mathbf{Q}_{p}, \forall \varepsilon>0$ справедливо неравенство

$$
\chi(A) \leq \inf \left\{\sum_{i=1} \chi\left(U_{i}\right): \bigcup_{i=1}^{\infty} U_{i} \supseteq A, \operatorname{diam}\left(U_{i}\right) \leq \varepsilon\right\},
$$

где все $U_{i}$ - открытые шары в $\mathbf{Q}_{p}$. С другой стороны, из свойства нормы (7) следует, что $\forall B \subset \mathbf{Q}_{p}$ c $\operatorname{diam}(B)=r \quad B$ лежит в открытом (и замкнутом одновременно) шаре $U=\left\{x:\left|x-x_{0}\right|_{p}^{\alpha} \leq r, x_{0} \in B\right\} \mathrm{c} \operatorname{diam}(U)=r$, а это означает вместе с равенством $\chi(U)=\operatorname{diam}^{1 / \alpha}(U)$, справедливым для всякого шара $U$, что $h_{\varepsilon}^{1 / \alpha}$ совпадает с правой частью неравенства (9), следовательно, $h^{1 / \alpha}\left(\mathbf{Z}_{p}\right) \geq \chi\left(\mathbf{Z}_{p}\right)$.

Рассмотрим $\mathbf{R} \times \mathbf{Z}_{p}$ как прямое произведение аддитивных групп и введем в этой группе метрику: фиксируем некоторое $\alpha>0$ и $\forall a, b \in \mathbf{R}$ и $\forall x, y \in \mathbf{Z}_{p}$ определим

$$
\hat{\rho}_{\alpha}((a, x),(b, y))=\max \left(|a-b|,|x-y|_{p}^{\alpha}\right) .
$$

Очевидно, что $\hat{\rho}_{\alpha}-$ инвариантная метрика, и топология, порождаемая ею, совпадает с топологией прямого произведения групп.

ОПреДЕЛЕНИЕ 2. Пусть $B$ обозначает подгруппу $\{(n,-n): n \in \mathbf{Z}\}$ группы $\mathbf{R} \times \mathbf{Z}_{p}$, тогда фактор-группа $\mathbf{T}_{p}=\left(\mathbf{R} \times \mathbf{Z}_{p}\right) / B$ называется $p$-адическим соленоидом $\left.{ }^{4}\right)$.

Можно показать [11], что $\mathbf{T}_{p}$ - связанная компактная абелева группа. Инвариантную и согласованную с топологией метрику $\rho_{\alpha}$ на $\mathbf{T}_{p}$ можно определить как фактор-метрику по стандартной схеме [11]: $\forall f, g \in \mathbf{T}_{p}$ положим

$$
\rho_{\alpha}(f, g)=\inf \left\{\hat{\rho}_{\alpha}((a, x),(b, y)):(a, x) \in f,(b, y) \in g\right\} .
$$

Следуюшая конструкция дает конкретную реализацию $\mathbf{T}_{p}$.

\footnotetext{
4) Это определение отличается от определения, данного в [11], там $B=\{(n, n): n \in \mathbf{Z}\}$, но т.к. отображение $x \mapsto-x$ есть автоморфизм аддитивной группы $\mathbf{Z}_{p}$, то соответствующие фактор-группы изоморффны.
} 
ТЕОРема 4. Рассмотрим произведение $[0,1) \times \mathbf{Z}_{p}$ и определим в нем сложсение по правилу: $\forall f=(\xi, x), \quad g=(\eta, y) \in[0,1) \times \mathbf{Z}_{p}$

$$
f+g=(\xi+\eta-[\xi+\eta], x+y+[\xi+\eta]),
$$

где $[\xi+\eta]-$ щелая часть вещественного числа $\xi+\eta$. Для некоторого фиксированного $\alpha>0$ зададим метрику $\rho_{\alpha}$, положив

$$
\rho_{\alpha}(f, g)=\min \left(\ell_{\alpha}(f-g), \ell_{\alpha}(g-f)\right)
$$

где $\ell_{\alpha}(f)=\max \left(\xi,|x|_{p}^{\alpha}\right)$. Тогда полученная таким образом абелева группа с топологией, индуцированной метрикой, алгебраически и изометрично изоморфна $\mathbf{T}_{p} c$ метрикой (11).

ДокАЗАТЕльство. Алгебраический изоморфизм устанавливается практически так же, как в доказательстве теоремы 10.15 из [11]. Из (10), (11) и определения подгруппы $B$ следует, что для метрики на $\mathbf{T}_{p}$ справедливо равенство

$$
\rho_{\alpha}(f, 0)=\inf \left\{\max \left(|\xi-n|,|x+n|_{p}^{\alpha}\right): n \in \mathbf{Z}\right\}
$$

но т.к. $\forall x \in \mathbf{Z}_{p}$ выполняется $|x+n|_{p}^{\alpha} \leq 1$, то в последней формуле можно оставить значения $n=0,1$. Теперь, учитывая, что $(-f)=(1-\xi,-x-1)$ при $\xi \neq 0$, легко убедиться в том, что метрики действительно совпадают.

Пользуясь теоремой 4 и тем, что $\forall x \in \mathbf{Z}_{p} \rho_{\alpha}((0, x), 0)=|x|_{p}^{\alpha}$ и $\{x\}_{p}=0$, а также тем, что множество $\left\{\{x\}_{p}: x \in \mathbf{Q}_{p}\right\}$ плотно в $[0,1)$ (в обычной топологии), можно показать что справедлива

Теорема 5. Отображсние $j: \mathbf{Q}_{p} \mapsto \mathbf{T}_{p}$, сопоставляющее каждому $x \in \mathbf{Q}_{p}$ әлемент $\left(\{x\}_{p},[x]_{p}\right) \in \mathbf{T}_{p}$, есть инвективный гомоморфизм аддитивной группьи $\mathbf{Q}_{p}$ в $\mathbf{T}_{p}$ и локальная изометрия из $\left(\mathbf{Q}_{p},|\cdot|_{p}^{\alpha}\right)$ в $\left(\mathbf{T}_{p}, \rho_{\alpha}\right) \quad \forall \alpha>0$. Кроме того, $\operatorname{Ran}(\mathbf{Q}) \subset \operatorname{Ran}\left(\mathbf{Q}_{p}\right)$ плотен в $\mathbf{T}_{p}$. Поскольку $\mathbf{T}_{p}$ полно (в силу компактности [12]), то $\mathbf{T}_{p}$ есть пополнение $\mathbf{Q}$ по метрике $\rho_{\alpha}{ }^{5)}$.

$p$-Адический соленоид $\mathbf{T}_{p}$ - компактная абелева группа [11], поэтому на нем существует единственная инвариантная конечная мера Хаара $\chi$. Покажем, что хаусдорфова мера $h_{\delta}$ на $\left(\mathbf{T}_{p}, \rho_{\alpha}\right)$ при $\delta=\alpha^{-1}+1$ совпадает (с точностью до конечного и ненулевого множителя) с $\chi$ на всех борелевских подмножествах $\mathbf{T}_{p}$. Поскольку $\rho_{\alpha}-$ инвариантная метрика, то достаточно показать, что для множества $U_{1 / 2}=\{y \in \mathbf{R} \times$ $\left.\mathbf{Z}_{p}: \rho_{\alpha}(y, 0)<1 / 2\right\} \quad h_{\delta}\left(\phi\left(U_{1 / 2}\right)\right) \stackrel{L}{\simeq} 1$, где $\phi$ обозначает каноническую проекцию $\mathbf{R} \times \mathbf{Z}_{p}$ на $\mathbf{T}_{p}$. Но поскольку $\forall a, b \in B \subset \mathbf{R} \times \mathbf{Z}_{p}$ имеем $\hat{\rho}_{\alpha}(a, b) \geq 1$ при $a \neq b$, то сужение $\phi$ на $U_{1 / 2}$ есть изометрия. Поэтому, используя равенство $U_{1 / 2}=\left(-\frac{1}{2}, \frac{1}{2}\right) \times p^{n_{\alpha}} \mathbf{Z}_{p}$ для некоторого $n_{\alpha}>0$, по теореме 2 получаем, что

$$
h_{\delta}\left(\phi\left(U_{1 / 2}\right)\right)=\hat{h}_{\delta}\left((-1 / 2,1 / 2) \times\left\{x \in \mathbf{Z}_{p}:|x|_{p} \leq p^{-1}\right\}\right) \stackrel{L}{\simeq} p^{-n_{\alpha}} \tilde{h}_{1 / \alpha}\left(\mathbf{Z}_{p}\right) .
$$

Здесь $\hat{h}_{\delta}$ и $\tilde{h}_{\delta}$ обозначают хаусдорфовы меры в $\mathbf{R} \times \mathbf{Z}_{p}$ и $\mathbf{T}_{p}$, соответственно. Таким образом, как и в случае с $\mathbf{Q}_{p}$, локальная и глобальная хаусдорфовы размерности $\left(\mathbf{T}_{p}, \rho_{\alpha}\right)$ совпадают и равнь $\alpha^{-1}+1$.

\footnotetext{
5) Заметим, что хотя и $j$ - не вложение $\left(\operatorname{Ran}\left(\mathbf{Q}_{p}\right)\right.$ плотно в $\left.\mathbf{T}_{p}\right)$, сужения $j$ на $p^{M} \mathbf{Z}_{p}$ суть $(L-)$ изометрии $\forall M \in \mathbf{N}(\mathbf{Z})$.
} 


\section{3. НЕПРЕРЫВНЫЕ ОТОБРАЖЕНИЯ $\mathbf{Q}_{p}$ В C}

Определим $\forall n \in \mathbf{Z}$ и $\forall m \in \overline{\mathbf{N}} \equiv \mathbf{N} \cup\{\infty\}$ комплекснозначные функции $\chi_{n}^{(m)}(\cdot)$ на $\mathbf{Q}_{p}$, положив $\forall x \in \mathbf{Q}_{p}$

$$
\chi_{n}^{(m)}(x)=\exp \left(\frac{i 2 \pi}{p} \sum_{k=0}^{m} x_{n-k} p^{-k}\right),
$$

где $x_{n}-n$-й коэффициент разложения $x$ в ряд (6). Нетрудно проверить, что $\chi_{n}^{(m)}(\cdot)$ непрерывны. Заметим, что $\chi_{n}^{(\infty)}(\cdot)$ совпадает с непрерывным аддитивным характером $\chi_{\frac{1}{p^{n+1}}}(\cdot)$ на $\mathbf{Q}_{p}[11,13]$.

ОПреДЕЛЕниЕ 3. Для каждого $s \in U_{1} \equiv\{z \in \mathbf{C}:|z|<1\}$ и $\forall m \in \overline{\mathbf{N}}$ определим непрерывное отображение $\Upsilon_{s}^{(m)}: \mathbf{Q}_{p} \mapsto \mathbf{C}$, положив $\forall x \in \mathbf{Q}_{p}$

$$
\Upsilon_{s}^{(m)}(x)=\frac{1-s^{v(x)}}{1-s}+\sum_{n=v(x)}^{\infty} s^{n} \chi_{n}^{(m)}(x)=\left[\Upsilon_{s}^{(m)}\right](x)+\left\{\Upsilon_{s}^{(m)}\right\}(x)
$$

Здесь $\left[\Upsilon_{s}^{(m)}\right](x)=\sum_{n=0}^{\infty} s^{n} \chi_{n}^{(m)}(x)-$ “целая часть" $\Upsilon_{s}^{(m)}(x)$ и $\left\{\Upsilon_{s}^{(m)}\right\}=\Upsilon_{s}^{(m)}(x)-$ $\left[\Upsilon_{s}^{(m)}\right](x)$ - “дробная часть" $\Upsilon_{s}^{(m)}(x)$.

Очевидно, что определение корректно, и формула (15) определяет голоморфиню в круге $U_{1}$ функцию от $s$ при каждом фиксированном $x \in \mathbf{Q}_{p}$. Кроме того, учитывая, что $\forall q \in \mathbf{N}(\mathbf{Q})$ коэфффициенты ряда (6) нулевые (периодичны [10]) начиная с некоторого $n_{q}$, можно показать, что имеет место следуюший любопытный факт: при $m<\infty$ $\Upsilon_{(\cdot)}^{(m)}(q)-\Upsilon_{(\cdot)}^{(m)}(0)$ есть полином (рациональная функция), а при $m=\infty$ функция $\varrho(\cdot) \cdot \Upsilon_{(\cdot)}^{(\infty)}(q)$ целая (мероморфная, т.е. отношение двух целых), где $\varrho(s)=\prod_{k=0}^{\infty}(1-$ $\left.p^{-k} s\right)$. Для $m<\infty$ доказательство последнего утверждения следует из периодичности $\chi_{n}^{(m)}(q)$ по $n$ при $n>n_{q}+m$; в случае $m=\infty \forall q \in \mathbf{Q}$ имеем $\chi_{n}^{(\infty)}(q)=$ $\exp \left(i 2 \pi\left\{q / p^{n+1}\right\}_{p}\right)=\exp \left(i 2 \pi q / p^{n+1}\right) \exp \left(-i 2 \pi\left[q / p^{n+1}\right]_{p}\right)$, поэтому аналитическое продолжение строится посредством разложения $\exp \left(i 2 \pi q / p^{n+1}\right)$ в ряд Тейлора с учетом периодичности $\exp \left(-i 2 \pi\left[q / p^{n+1}\right]_{p}\right)$ по $n$ при $n>n_{q}{ }^{6}$.

Нетрудно проверить, что $\forall x \in \mathbf{Q}_{p}$ имеют место соотношения

$$
\begin{gathered}
\left\{\Upsilon_{s}^{(m)}\right\}(x)=\left\{\Upsilon_{s}^{(m)}\right\}\left(\{x\}_{p}\right), \quad \Upsilon_{s}^{(m)}\left([x]_{p}\right)=\left[\Upsilon_{s}^{(m)}\right]\left([x]_{p}\right) \\
{\left[\Upsilon_{s}^{(m)}\right]\left(p^{-m} x\right)=\left[\Upsilon_{s}^{(m)}\right]\left(p^{-m}[x]_{p}\right)}
\end{gathered}
$$

Справедливо следуюшее основное свойство отображения $\Upsilon_{s}^{(m)}-$ скейлинг:

$$
\forall x \in \mathbf{Q}_{p} \quad \Upsilon_{s}^{(m)}(p x)=s \Upsilon_{s}^{(m)}(x)+1=p^{-\frac{1}{D_{s}}} e^{i \arg (s)} \Upsilon_{s}^{(m)}(x)+1,
$$

${ }^{6)}$ Аналитические свойства $\Upsilon_{(\cdot)}^{(m)}(x)$ для $x \in \mathbf{Q}_{p} \backslash \mathbf{Q}$ автору не известны, однако если в разложении (6), например, для $x \in \mathbf{Q}_{2}$ положить $x_{n}=\sum_{k=0}^{\infty} \delta_{n, 2^{k}}$, то в этом случае $\Upsilon_{s}^{(0)}(x)=$ $-2 \sum_{k=0}^{\infty} s^{2^{k}}$ и можно показать [14], что $U_{1}$ есть область голоморфности для $\Upsilon_{(\cdot)}^{(0)}(x)$. 
где $D_{s}=-\log _{p}^{-1}(|s|)$ будем называть скейлинговой размерностью $\Upsilon_{s}^{(m)}$. Это соотношение вытекает из того, что $\chi_{n+k}^{(m)}\left(p^{k} x\right)=\chi_{n}^{(m)}(x) \forall k \in \mathbf{Z}$.

Более того, используя (16) и (17), можно показать, что $\forall m<\infty$ множество $\operatorname{Ran} \Upsilon_{s}^{(m)}$ самоподобно. Точнее, пусть $B_{l}^{n} \equiv \Upsilon_{s}^{(m)}\left(\left\{x \in \mathbf{Q}_{p}:|x-l|_{p} \leq p^{-n}\right\}\right)$, тогда

$$
B_{l}^{n}=\bigcup_{\bar{l}=0}^{p^{m}-1}\left\{z_{l, \bar{l}}^{n}+e^{i \arg (s) m n} p^{-\frac{m n}{D_{s}}} B_{\bar{l}}^{0}\right\}
$$

$\forall n \in \mathbf{Z}, l \in \mathbf{Q}_{p}$. Здесь $z_{l, \bar{l}}^{n}-$ сдвиги в $\mathbf{C}$, зависящие от $l, \bar{l}$ и $n$. Таким образом, любое $B_{l}^{n}$ может быть получено из $p^{m}$ множеств $B_{\bar{l}}^{0}$ при помоши непрерывных движений плоскости $\mathbf{C}$ (сдвигов и поворотов) и масштабного преобразования.

Выясним теперь условия, при которых отображения $\Upsilon_{s}^{(m)}$ становятся вложениями. Для этого определим число

$$
\Delta_{s}^{(m)}=\inf \left\{\left|\Upsilon_{s}^{(m)}(x)-\Upsilon_{s}^{(m)}(y)\right|: \forall x, y \in \mathbf{Q}_{p}:|x-y|_{p}=1\right\}
$$

Используя то, что $\left|\chi_{0}^{(m)}(x)-\chi_{0}^{(m)}(y)\right| \geq 2 \sin (\pi / p)$ при $|x-y|_{p}=1$, можно получить следуюшую оценку:

$$
\frac{\Delta_{s}^{(m)}}{2} \geq \sin \left(\frac{\pi}{p}\right)-\frac{|s|}{1-|s|} .
$$

Отсюда получаем, что $\Delta_{s}^{(m)}>0$ при $|s|<s_{0}=\sin (\pi / p) /(1+\sin (\pi / p))$.

ТЕОРема 6. Пусть s и $m$ такие, что $\Delta_{s}^{(m)}>0$, тогда $\Upsilon_{s}^{(m)}-L$-изометрия из $\left(\mathbf{Q}_{p},|\cdot|_{p}^{D_{s}^{-1}}\right)$ в $(\mathbf{C},|\cdot|)$ и, следовательно, $\Upsilon_{s}^{(m)}-$ вложсение $e^{7)}$.

ДокАЗАТЕльСтво получается из следующей оценки, вытекаюшей из (17), и определения (14): $\forall x, y \in \mathbf{Q}_{p}$

$$
\Delta_{s}^{(m)}|s|^{v(x-y)} \leq\left|\Upsilon_{s}^{(m)}(x)-\Upsilon_{s}^{(m)}(y)\right| \leq \frac{2}{1-|s|}|s|^{v(x-y)}
$$

Из последней теоремы и теоремы 1 получаем, что если $\Delta_{s}^{(m)}>0$, то локальная $u$ глобальная хаусдорфовы размерности $\Upsilon_{s}^{(m)}\left(\mathbf{Q}_{p}\right)$ равны $D_{s}$. Из теорем 1 и 6 также вытекает, что $h^{D_{s}}\left(\Upsilon_{s}^{(m)}\left(\mathbf{Z}_{p}\right)\right) \stackrel{L}{\simeq} 1$. Поэтому мы можем ввести меру $\mu_{f}$ на $\mathbf{Q}_{p}$, положив $\forall B \subset \mathbf{C}$

$$
\mu_{f}(B)=\frac{h^{\delta}\left(B \cap \Upsilon_{s}^{(m)}\left(\mathbf{Q}_{p}\right)\right)}{h^{\delta}\left(\Upsilon_{s}^{(m)}\left(\mathbf{Z}_{p}\right)\right)} .
$$

Сужение этой меры на $\Upsilon_{s}^{(m)}\left(\mathbf{Z}_{p}\right)$ совпадает с мультифрактальной мерой $\mu_{f}^{0}[15]$ множества $\Upsilon_{s}^{(m)}\left(\mathbf{Z}_{p}\right)$, кроме того, оказывается справедливой следуюшая

\footnotetext{
${ }^{7)}$ Можно показать, используя компактность $\mathbf{Z}_{p}$, свойство (17) и полноту $\mathbf{Q}_{p}$ и $\mathbf{C}$, что если $\Upsilon_{s}^{(m)}$ - инъективное отображение, то оно есть вложение.
} 
ТЕОрема 7. Пусть $\chi(\cdot)$ - стандартная мера Хаара в $\mathbf{Q}_{p}$, тогда если $\Delta_{s}^{(m)}>0$, mo

$$
\mu_{f}(\cdot)=\chi\left(\left(\Upsilon_{s}^{(m)}\right)^{-1}(\cdot)\right)
$$

ДокАЗАТЕЛЬСтво. Поскольку $\Upsilon_{s}^{(m)}$ - вложение, то кластеры $B_{l}^{n}$, определенные в $(18)$, не пересекаются, и поэтому при $m<\infty$ из самоподобия множества $\Upsilon_{s}^{(m)}\left(\mathbf{Q}_{p}\right)$ следует, что $h^{D_{s}}\left(\Upsilon_{s}^{(m)}(B)\right)=h^{D_{s}}\left(\Upsilon_{s}^{(m)}\left(\mathbf{Z}_{p}\right)\right) \chi(B)$ для всех открытых В. Доказательство теоремы в случае $m=\infty$ вытекает из теоремы 1 и следующей леммы.

Лемма 1. Определим на $\mathbf{Q}_{p}$ псевдометрики $\rho_{m}$, положив $\forall x, y \in \mathbf{Q}_{p}$

$$
\rho_{m}(x, y)=\left|\Upsilon_{s}^{(m)}(x)-\Upsilon_{s}^{(m)}(y)\right|
$$

и пусть $\kappa_{m, \infty}-$ расхождение $\rho_{m} u \rho_{\infty}$, тогда

$$
\kappa_{m, \infty}<\frac{4 \pi}{(1-|s|)\left(\Delta_{s}^{(m)}+\Delta_{s}^{(\infty)}\right)} p^{-m}
$$

ДокАЗАТЕЛЬСтво леммы. Заметим, что благодаря скейлингу (17) в формуле (4) можно ограничиться $\sup$ по $x, y$ таким, что $|x-y|_{p}=1$, поэтому знаменатель в (4) больше или равен $\Delta_{s}^{(m)}+\Delta_{s}^{(\infty)}$. С другой стороны, поскольку $\forall x \in \mathbf{Q}_{p}$

$$
\left|\chi_{n}^{(\infty)}(x)-\chi_{n}^{(m)}(x)\right|=\left|1-\exp \left(\frac{i 2 \pi}{p} \sum_{k=m+1}^{\infty} x_{n-k} p^{-k}\right)\right|<2 \pi p^{-m}
$$

нетрудно показать, что числитель в (4) всегда меньше $4 \pi p^{-m} /(1-|s|)$.

Таким образом, теорема 7 позволяет при вычислении интегралов по фрактальной мере $\mu_{f}$ или $\mu_{f}^{0}$ пользоваться техникой интегрирования в $\mathbf{Q}_{p}[1]$. Например, можно вычислить интегралы по $\mu_{f}^{0}$ от любых полиномов от $z$ и $\bar{z}$ для $z \in \mathbf{C}$. Действительно, можно показать, что имеет место следуюшая формула:

$$
\begin{aligned}
\left\langle z^{L} \bar{z}^{\bar{L}}\right\rangle \equiv \int_{\mathbf{C}} z^{L} \bar{z}^{\bar{L}} d \mu_{f}^{0}(x) & =\int_{\mathbf{z}_{p}}\left(\Upsilon_{s}^{(m)}(x)\right)^{L}\left(\overline{\Upsilon_{s}^{(m)}(x)}\right)^{\bar{L}} d \chi(x)= \\
& =\sum_{n, \bar{n}=0}^{\infty} C_{n, \bar{n}}^{L, \bar{L}} s^{n} \bar{s}^{\bar{n}}
\end{aligned}
$$

При этом $C_{n, \bar{n}}^{L, \bar{L}} \in \mathbf{N}$ для $m=0, \infty$. В частности, для $m=\infty C_{n, \bar{n}}^{L, \bar{L}}$ есть число способов представить $n=n_{0}+\cdots+n_{L}$ и $\bar{n}=\bar{n}_{0}+\cdots+\bar{n}_{\bar{L}}$ так, чтобы $\sum_{k=0}^{L} p^{-n_{k}}=\sum_{k=0}^{\bar{L}} p^{-\bar{n}_{k}}$ $\bmod (p)$. Пользуясь этой формулой, можно получить, например, что $\left\langle z^{p}\right\rangle=\left\langle\bar{z}^{p}\right\rangle=1$ и $\left\langle z^{L} \bar{z}^{\bar{L}}\right\rangle=\left(1-|s|^{2}\right)^{-L} \delta_{L, \bar{L}}$ при $L, \bar{L}<p$.

Оставив в стороне вопросы строгого математического обоснования, укажем еще одно возможное применение теоремы 7. Рассмотрим квантовую частицу на фрактале $\mathbf{F}=$ 
$\operatorname{Ran}\left(\Upsilon_{s}^{(m)}\right)$, тогда $L^{2}\left(\mathbf{C}, \mu_{f}\right)$ можно принять за гильбертово пространство. Предположим, что уравнение Шредингера для собственных значений энергии $E$ можно записать в следующем виде ${ }^{8)}$ :

$$
E \Psi(z)=\int K(|z-\dot{z}|) \Psi(\dot{z}) d \mu_{f}(\dot{z}) .
$$

При помоши теоремы 7 мы можем перейти к эквивалентному уравнению на $L^{2}\left(\mathbf{Q}_{p}, \chi\right)$ с ядром $K\left(\left|\Upsilon_{s}^{(m)}(x)-\Upsilon_{s}^{(m)}(y)\right|\right)$ для $x, y \in \mathbf{Q}_{p}$. Очевидно, что в обшем случае это не дает ничего нового, ядро $K$ трансляционно-инвариантно в $\mathbf{C}$, но не инвариантна $\mu_{f}$ и, наоборот, в $\mathbf{Q}_{p}$ инвариантна $\chi$, но не инвариантно $K$. Однако, пользуясь $(17)$, можно представить ядро в виде

$$
K\left(|x-y|_{p}^{\alpha} \cdot\left|\left(\chi_{v(x-y)}^{(m)}(x-y)-1\right)-O^{x, y}(s)\right|\right),
$$

причем $\left|O^{x, y}(s)\right| \leq|s| /(1-|s|) \forall x, y \in \mathbf{Q}_{p}$. Таким образом, при $|s| \ll 1$ ядро зависит только от $x-y$, и поэтому преобразование $\Phi$ урье на $\mathbf{Q}_{p}[1,13]$ диагонализует гамильтониан. Кроме того, при $p=2,3$, пользуясь тем, что $\left|\chi_{v(x)}^{(m)}(x)-1\right|=2 \sin (\pi / p)$, можно показать, что спектр гамильтониана (в этом приближении) имеет вид $\left\{E_{n}=\widetilde{K}\left(p^{n}\right): n \in\right.$ $\mathbf{Z}\}$ и все состояния, кроме основного, строго локализованы (т.е. собственные функции имеют компактный носитель [1]). Здесь $\widetilde{K}(\cdot)$ - фурье-образ функции $K\left(2 \sin (\pi / p)|\cdot| \begin{array}{l}\alpha \\ p\end{array}\right)$. Используя формулы (14), (15) и технику $p$-адического интегрирования, последовательно разлагая (27) в ряд по степеням $s$ (при фиксированном $\alpha$ ), можно найти фурье-образы коэффициентов ряда по крайней мере в случае $m=\infty$ и $p=2,3$. Таким образом возникает возможность вычислить дальнейшие поправки к спектру и волновым функциям по теории возмушений. Заметим, что вышеизложенное применимо в равной степени и к задаче диффузии на фрактале $\mathbf{F}$.

\section{4. НЕПРЕРЫВНЫЕ ОТОБРАЖЕНИЯ $\mathbf{T}_{p} \mathbf{B ~ R}^{3}$}

Определим $\forall n \in \mathbf{Z}$ и $\forall m \in \overline{\mathbf{N}} \equiv \mathbf{N} \cup\{\infty\}$ непрерывные комплекснозначные функции $\tilde{\chi}_{n}^{(m)}(\cdot)$ на $\mathbf{R} \times \mathbf{Z}_{p}$, положив $\forall(\xi, x) \in \mathbf{R} \times \mathbf{Z}_{p}$

$$
\tilde{\chi}_{n}^{(m)}(\xi, x)=\exp \left(i 2 \pi \xi \frac{\theta\left(p^{m-n}-|x|_{p}\right)}{p^{\min (n, m)+1}}\right) \chi_{n}^{(m)}(x) .
$$

Здесь функция Хевисайда $\theta(\cdot)$ есть индикатор множества $[0, \infty]$. Нетрудно проверить, что для функций $\tilde{\chi}_{n}^{(m)}(\cdot)$ выполнено следуюшее соотношение:

$$
\tilde{\chi}_{n}^{(m)}(\xi+l, x-l)=\tilde{\chi}_{n}^{(m)}(\xi, x)
$$

$\forall l \in \mathbf{Z}, \quad(\xi, x) \in \mathbf{R} \times \mathbf{Z}_{p}$, т.е. они постоянны на классах смежности групшы $B$ (см. определение 2). Отметим, что $\forall f=(\xi, x) \in \mathbf{T}_{p} \quad \tilde{\chi}_{n}^{(\infty)}(\xi, x)=\tilde{\chi}_{\left(-p^{-n-1}\right)}(f)$, где $\tilde{\chi}_{(q)}(\cdot)-$ непрерывные адлитивные характеры на $\mathbf{T}_{p}[11]$ (с учетом замечания 4$)$.

\footnotetext{
8) Такую квантовую систему можно мыслить себе как электрон на плоскости, находящийся в глубоком потенциале, сосредоточенном на фрактале $\mathbf{F}$. Тогда в пределе сильной связи можно считать, что волновая функция практически полностью локализована на $\mathbf{F}$ и ядро $K$ определяется интегралами типа интегралов перекрытия (см., например, [6]).
} 
ОПРЕДЕЛЕниЕ 4. Для каждого $s \in U_{1}$ и $\forall m \in \overline{\mathbf{N}}$ определим непрерывное отображение $\omega_{s}^{(m)}: \mathbf{R} \times \mathbf{Z}_{p} \mapsto \mathbf{C}$, положив $\forall(\xi, x) \in \mathbf{R} \times \mathbf{Z}_{p}$

$$
\omega_{s}^{(m)}(\xi, x)=\sum_{n=0}^{\infty} s^{n} \tilde{\chi}_{n}^{(m)}(\xi, x) .
$$

Заметим, что $\forall x \in \mathbf{Z}_{p}$ имеет место равенство

$$
\Upsilon_{s}^{(m)}(x)=\omega_{s}^{(m)}(0, x)
$$

Введем отображение $\sigma_{a}: \mathbf{S}_{1} \times \mathbf{C} \mapsto \mathbf{R}^{3}$ для некоторого $a \in \mathbf{C}$, задав соответствие $\mathbf{S}_{1} \times$ $\mathbf{C} \ni\left(e^{i 2 \pi \xi}, z\right) \mapsto\left(x_{1}, x_{2}, x_{3}\right) \in \mathbf{R}^{3}$ по формулам

$$
x_{1}+i x_{3}=e^{i 2 \pi \xi}|a|\left(1+\operatorname{Re}\left(\frac{z}{a}\right)\right), \quad x_{2}=|a| \operatorname{Im}\left(\frac{z}{a}\right),
$$

где $\mathbf{S}_{1}$ - единичная окружность в $\mathbf{C}$. Теперь отображение из $\mathbf{T}_{p}$ в $\mathbf{R}^{3}$ определим как композицию $\sigma_{a}$ и отображения из $\mathbf{T}_{p}$ в $\mathbf{S}_{1} \times \mathbf{C}$, которое мы сейчас построим. С этой целью будем рассматривать $\mathbf{T}_{p}$ и $\mathbf{S}_{1} \times \mathbf{C}$ как фактор-пространства относительно действий группы $\mathbf{Z}$ на $\mathbf{R} \times \mathbf{Z}_{p}$ и $\mathbf{R} \times \mathbf{C}$, а именно: $\forall n \in \mathbf{Z}$ действие на $\mathbf{R} \times \mathbf{Z}_{p}$ есть сдвиг на элемент $(n,-n) \in B$ и на $\mathbf{R} \times \mathbf{C}-$ сдвиг на элемент $(n, 0) \in \mathbf{Z} \times\{0\}$. Пусть $\otimes \omega_{s}^{(m)} \equiv i d \times \omega_{s}^{(m)}: \mathbf{R} \times$ $\mathbf{Z}_{p} \mapsto \mathbf{R} \times \mathbf{C}$, где $i d$ - тождественное отображение. Нетрудно увидеть, что свойство (29) гарантирует сушествование непрерывного фактор-отображения $\mho_{s}^{(m)}: \mathbf{T}_{p} \mapsto \mathbf{S}_{1} \times \mathbf{C}$ и вместе с ним отображения $\Omega_{s, a}^{(m)}: \mathbf{T}_{p} \mapsto \mathbf{R}^{3}$ таких, что следуюшая диаграмма

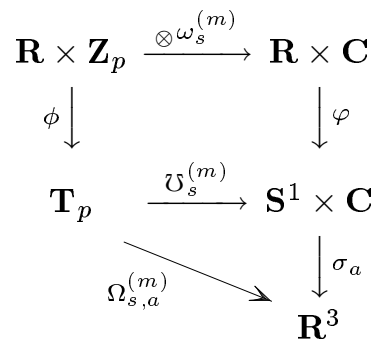

коммутативна. Здесь $\phi$ и $\varphi$ - соответствуюшие канонические проекции. Более того, можно показать, что действия группы $\mathbf{Z}$ на $\mathbf{R} \times \mathbf{Z}_{p}$ и $\mathbf{R} \times \mathbf{C}$ согласованы в том смысле, что $\forall f \in \mathbf{T}_{p}$ имеет место соотношение

$$
\otimes \omega_{s}^{(m)}\left(\phi^{-1}(f)\right)=\varphi^{-1}\left(\mho_{s}^{(m)}(f)\right) .
$$

Введем инвариантную метрику $\hat{d}$ в $\mathbf{R} \times \mathbf{C}$ такую, что $\hat{d}\left(\left(\xi_{1}, z_{1}\right),\left(\xi_{2}, z_{2}\right)\right)=$ $\max \left(\left|\xi_{1}-\xi_{2}\right|,\left|z_{1}-z_{2}\right|\right) \quad \forall\left(\xi_{1}, z_{1}\right),\left(\xi_{2}, z_{2}\right) \in \mathbf{R} \times \mathbf{C}$. B $\mathbf{S}_{1} \times \mathbf{C}$ метрику $d$ определим как фактор-метрику метрики $\hat{d}$ по той же схеме, что и в (11). Тогда нетрудно получить, что $\forall\left(e^{i 2 \pi \xi_{1}}, z_{1}\right),\left(e^{i 2 \pi \xi_{2}}, z_{2}\right) \in \mathbf{S}_{1} \times \mathbf{C}$ (полагая при этом, что $\left.\xi_{1}, \xi_{2} \in[0,1)\right)$

$$
d\left(\left(e^{i 2 \pi \xi_{1}}, z_{1}\right),\left(e^{i 2 \pi \xi_{1}}, z_{2}\right)\right)=\max \left(\inf _{n=0, \pm 1}\left(\left|\xi_{1}-\xi_{2}+n\right|\right),\left|z_{1}-z_{2}\right|\right) .
$$


Поскольку $\phi, \varphi$ и сужение $\sigma_{a}$ на любое ограниченное множество в $\mathbf{S}_{1} \times \mathbf{C}$ суть $L$-сжатия, то если мы покажем, что $\otimes \omega_{s}^{(m)}-L$-сжатие, тогда $L$-сжатиями будут $\mho_{s}^{(m)}$ и $\Omega_{s, a}^{(m)}$. Более того, если $\otimes \omega_{s}^{(m)}-L$-изометрия, то $\mho_{s}^{(m)}$ - также $L$-изометрия. Действительно, используя (33), можно показать, что $\forall f, g \in \mathbf{T}_{p}$ найдутся такие $(\xi, x) \in f,(\eta, y) \in g$, что $\forall \varepsilon>0$ вьполнена цепочка неравенств

$$
\rho_{\alpha}(f, g) \leq \hat{\rho}_{\alpha}((\xi, x),(\eta, y)) \stackrel{L}{\preceq} \hat{d}\left({ }_{\otimes} \omega_{s}^{(m)}(\xi, x), \otimes \omega_{s}^{(m)}(\eta, y)\right) \leq d\left(\mho_{s}^{(m)}(f), \mho_{s}^{(m)}(g)\right)+\varepsilon .
$$

Очевидно также, что если сужение $\sigma_{a}$ на $\operatorname{Ran}\left(\varphi{ } \otimes \omega_{s}^{(m)}\right)$ является $L$-изометрией, то отображение $\Omega_{s, a}^{(m)}$ также будет $L$-изометрией.

Для того чтобы выяснить условия, при которых $\otimes \omega_{s}^{(m)}-L$-сжатие или $L$-изометрия, введем следующие два числа:

$$
\begin{aligned}
& \tilde{\Delta}_{s}^{m}=\inf _{x, y \in \mathbf{Z}_{p}, \xi \in \mathbf{R}}\left(\left|s^{v(x-y)}\left(\omega_{s}^{(m)}(\xi, x)-\omega_{s}^{(m)}(\xi, y)\right)\right|\right), \\
& \gamma_{s, a}^{m}=-\inf _{(\xi, x) \in \mathbf{R} \times \mathbf{Z}_{p}}\left(\operatorname{Re}\left(\omega_{s}^{(m)}(\xi, x) / a\right)\right) .
\end{aligned}
$$

Нетрудно проверить, что для $\tilde{\Delta}_{s}^{m}$ имеет место та же оценка, что и в $(20)$, таким образом, $\tilde{\Delta}_{s}^{m}>0$ при $|s|<s_{0}$. Кроме того, можно показать, что $\tilde{\Delta}_{s}^{\infty}=\Delta_{s}^{\infty}$ (см. ниже). Теперь упомянутые выше условия вытекают из следуюшей цепочки неравенств:

$$
\begin{aligned}
\tilde{\Delta}_{s}^{m} \max \left(|\xi-\eta|,|x-y|_{p}^{\frac{1}{D_{s}}}\right) & \stackrel{L}{\prec} \max \left(|\xi-\eta|, \Delta_{s}^{m}|x-y|_{p}^{\frac{1}{D_{s}}}-\frac{2 \pi}{p(1-|s|)}|\xi-\eta|\right) \leq \\
& \leq \max \left(|\xi-\eta|,\left|\omega_{s}^{(m)}(\xi, x)-\omega_{s}^{(m)}(\eta, y)\right|\right) \leq \\
& \leq \max \left(|\xi-\eta|, \frac{1}{(1-|s|)}\left(|x-y|_{p}^{\frac{1}{D_{s}}}+\frac{2 \pi}{p}|\xi-\eta|\right)\right) \preceq \\
& \stackrel{L}{\preceq} \max \left(|\xi-\eta|,|x-y|_{p}^{\frac{1}{D_{s}}}\right),
\end{aligned}
$$

первое и последнее из которых - следствие соотношений (3), остальные получаются по аналогии с (21). Из элементарных геометрических соображений следует, что сужение $\sigma_{a}$ на любое компактное множество, лежашее в $\Pi_{a} \equiv\{z \in \mathbf{C}: \operatorname{Re}(z / a)>1\}$, есть $L$-изометрия, но при $\gamma_{s, a}^{m}<1 \quad \operatorname{Ran}\left(\mho_{s}^{(m)}\right) \subset \Pi_{a}$. Таким образом, доказана

ТеОрема 8. Пусть $\hat{\rho}_{\alpha}, \rho_{\alpha}, \hat{d}, d$ - соответствующие метрики в $\mathbf{R} \times \mathbf{Z}_{p}, \mathbf{T}_{p}$, $\mathbf{R} \times \mathbf{C}, \mathbf{S}_{1} \times \mathbf{C}, \quad$ в $\mathbf{R}^{3}$ метрика стандартная евклидова, и пусть $\alpha=D_{s}^{-1}$. Тогда для любих $m \in \overline{\mathbf{N}} u s \in U_{1}$

1) $\otimes \omega_{s}^{(m)}-L$-сжсатие $и$, следовательно, $\mho_{s}^{(m)}$ u $\Omega_{s, a}^{(m)}-$ также L-сжатия;

2) если $\tilde{\Delta}_{s}^{m}>0$, mо $\otimes \omega_{s}^{(m)}$ и $\mho_{s}^{(m)}-L$-изометрии, если $\kappa$ тому же $\gamma_{s, a}^{m}<1$, то $\Omega_{s, a}^{(m)}-$ L-изометрия.

Отсюда немедленно получаем, что при s u a maкux, чmo $\tilde{\Delta}_{s}^{m}>0, \gamma_{s, a}^{m}<1$, отобрахение $\Omega_{s, a}^{(m)}$ есть непрерьвное вложсение $\mathbf{T}_{p}$ в $\mathbf{R}^{3}$, локальная и глобальная хаусдорфовы размерности $\Omega_{s, a}^{(m)}\left(\mathbf{T}_{p}\right)$ равны $D_{s}+1$. 
Опишем теперь геометрическую структуру множества $\Omega_{s, a}^{(m)}\left(\mathbf{T}_{p}\right)$. Для этого заметим, что $\left(\mathbf{T}_{p}, \rho_{\alpha}\right)$ можно рассматривать как тотальное пространство локально-тривиального расслоения $\left(\mathbf{T}_{p}, \mathbf{S}_{1}\right)$ с проекцией $\chi_{0}: \mathbf{T}_{p} \mapsto \mathbf{S}_{1}$ такой, что $\chi_{0}(\xi, x)=$ $\exp (i 2 \pi \xi) \in \mathbf{S}_{1} \quad \forall(\xi, x) \in \mathbf{T}_{p} \quad$ (очевидно, что $\chi_{0}$ непрерывно, т.к. это характер на $\left.\mathbf{T}_{p}\right)$, и слоями $\chi_{0}^{-1}(\exp (i 2 \pi \xi))=\left(\xi, \mathbf{Z}_{p}\right)$, изометричными $\left(\mathbf{Z}_{p},\left.|\cdot|\right|_{p} ^{\alpha}\right)$. Нетрудно увидеть, что отображение $\mho_{s}^{(m)}$ есть послойный морфизм расслоения $\left(\mathbf{T}_{p}, \mathbf{S}_{1}\right)$ в тривиальное расслоение $\left(\mathbf{S}_{1} \times \mathrm{D}\left(r_{s}\right), \mathbf{S}_{1}\right) \subset\left(\mathbf{S}_{1} \times \mathbf{C}, \mathbf{S}_{1}\right)$ (где $\mathrm{D}\left(r_{s}\right)$ - замкнутый диск радиуса $\left.r_{s}=(1-|s|)^{-1}\right)$, при этом слои суть множества $\omega_{s}^{(m)}\left(\xi, \mathbf{Z}_{p}\right) \subset \mathrm{D}\left(r_{s}\right)$ для каждого фиксированного $\xi$, и если $\mho_{s}^{(m)}-L$-изометрия, то эти множества - фракталы с глобальными и локальными размерностями, равными $D_{s}$. Более того, из (31) следует, что слой с $\xi=0$ изометричен $\Upsilon_{s}^{(m)}\left(\mathbf{Z}_{p}\right)$. Положим для простоты, что $a>r_{s}$, тогда $\sigma_{a}$ есть стандартное вложение $\left(\mathbf{S}_{1} \times \mathrm{D}\left(r_{s}\right), \mathbf{S}_{1}\right)$ в полноторие (тор вместе со своей внутренностью) в $\mathbf{R}^{3}$, причем слои изометрично переходят в диски, лежашие в плоскости, повернутой вокруг оси $x_{2}$ на угол $2 \pi \xi$ относительно плоскости $x_{3}=0$.

Определим действие элемента $t$ группы $\mathbf{R}$ на $f \in \mathbf{T}_{p}$ как сдвиг $f \mapsto f_{t}=f+\phi(t, 0)$ и рассмотрим орбиты этой группы в $\mathbf{T}_{p}$. Можно показать [11], что каждая орбита есть плотное множество в $\mathbf{T}_{p}$. Поскольку отображения $\mho_{s}^{(m)}$ и $\Omega_{s, a}^{(m)}-L$-сжатия, то образы этих орбит суть непрерывные кривые, которые также плотно наматываются на $\mho_{s}^{(m)}\left(\mathbf{T}_{p}\right) \subset \mathbf{S}_{1} \times \mathrm{D}\left(r_{s}\right)$ и $\Omega_{s, a}^{(m)} \subset \mathbf{R}^{3}$, соответственно.

Оказывается, что в случае $m=\infty$ образы орбит группы $\mathbf{R}$ суть гладкие кривые, которые являются траекториями некоторой динамической системы.

ТЕОрема 9. Пусть $\Omega_{s, a}^{(\infty)}-L$-изометрия, тогда на всем $\mathbf{R}^{3}$ существует липшицево векторное поле $\Gamma: \mathbf{R}^{3} \mapsto \mathbf{R}^{3}$ такое, что $\forall f \in \mathbf{T}_{p}$

$$
\Gamma\left(\Omega_{s, a}^{(\infty)}(f)\right)=-2 \pi\left(L_{(2)} \Omega_{s, a}^{(\infty)}(f)+p^{-1} \operatorname{Re}\left(\tilde{\chi}_{-1}^{(\infty)}(f)\left(L_{(3)}+i L_{(1)}\right)\right) \Omega_{s / p,+0 a}^{(\infty)}(f)\right)
$$

Действие однопараметрической группь гомеоморфизмов $U_{t}: \mathbf{R}^{3} \mapsto \mathbf{R}^{3}$, порождаемой уравнением

$$
\frac{d}{d t} r=\Gamma(r)
$$

согласовано с действием $\mathbf{R}$ в $\mathbf{T}_{p}$ в том смицсле, что $\forall f \in \mathbf{T}_{p}, \forall t \in \mathbf{R}$

$$
\Omega_{s, a}^{(\infty)}\left(f_{t}\right)=U_{t} \Omega_{s, a}^{(\infty)}(f)
$$

Здесь $\left(L_{(k)}\right)_{i, j}=\varepsilon_{i, j, k}$, где $\varepsilon_{i, j, k}$ - символ Леви-Чивитьи и $\Omega_{s / p,+0 a}^{(\infty)}(f) \equiv$ $\lim _{\varepsilon \rightarrow+0} \Omega_{s / p, \varepsilon a}^{(\infty)}(f)$.

ДокаЗАТЕльство. Покажем сначала, что $\Gamma_{\mid \operatorname{Ran}\left(\Omega_{s, a}^{(\infty)}\right)}-L$-сжатие. Нетрудно убедиться, что для этого достаточно показать, что $\left(\left(\Omega_{s, a}^{(\infty)}\right)^{-1} \circ \Omega_{s / p,+0 a}^{(\infty)}\right)_{\mid \operatorname{Ran}\left(\Omega_{s, a}^{(\infty)}\right)}$ есть $L$-сжатие, но это непосредственно следует из того, что $\left(\Omega_{s, a}^{(\infty)}\right)^{-1}$ и $\Omega_{s / p,+0 a}^{(\infty)}-L$-сжатия для $\left(\mathbf{T}_{p}, \rho_{\alpha}\right)$ при $\alpha=D_{s}^{-1}\left(\Omega_{s / p,+0 a}^{(\infty)}-L\right.$-сжатие, т.к. $\left.\rho_{\alpha+1} \stackrel{L}{\preceq} \rho_{\alpha}\right)$. Теперь сушествование липшицева отображения $\Gamma$ на всем $\mathbf{R}^{3}$, удовлетворяюшего (37), гарантируется 

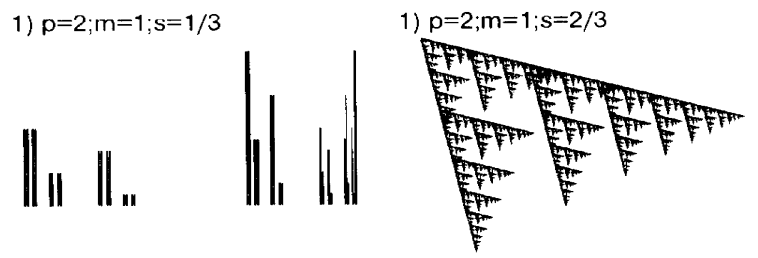

2) $p=2 ; m=\infty ; s=2 / 3$
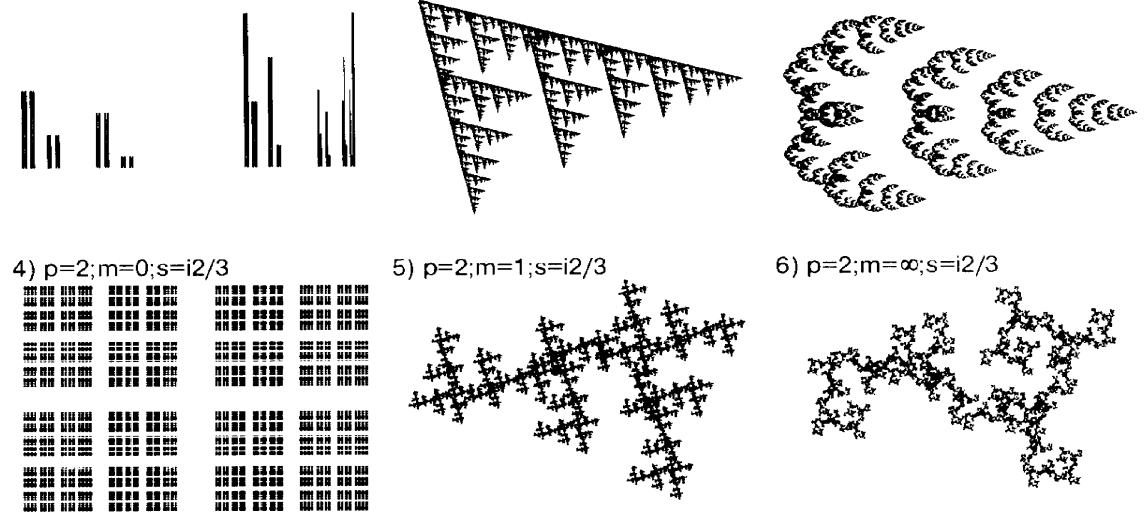

3) $p=2 ; m=\infty ; s=-2 / 3$

7) $p=2 ; m=0 ; s=2 / 3 \exp (i \pi / 6)$

6) $p=2 ; m=\infty ; s=i 2 / 3$
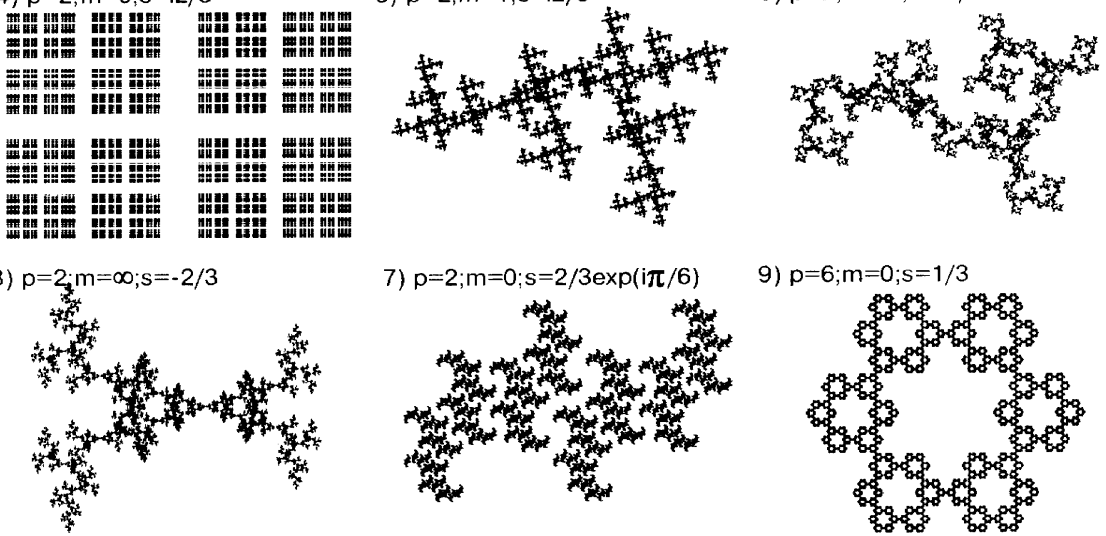

10) $p=3 ; m=0 ; s=1 / 2$
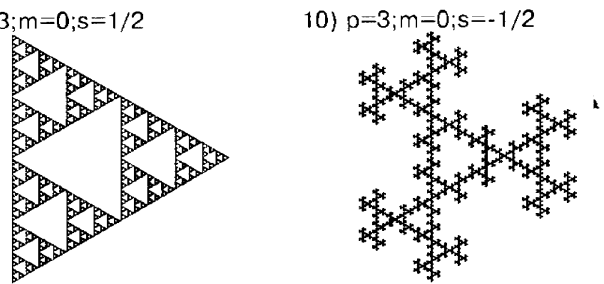

9) $p=6 ; m=0 ; s=1 / 3$

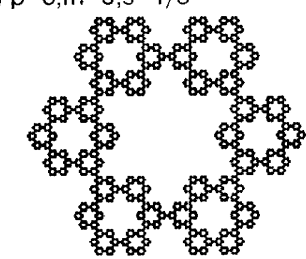

12) $p=3 ; m=\infty ; s=.46$

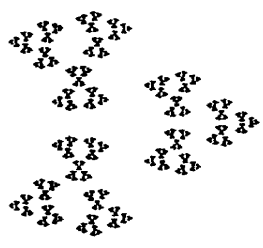

Рис. 1. Образы $\mathbf{Z}_{p}$ в комплексной плоскости при различных $p, m$ и $s$.

теоремой Кирсбрауна [8]. Непосредственно из формул (28), (30) вытекает, что $\forall t \in \mathbf{R}$, $x \in \mathbf{Z}_{p}$ имеет место равенство

$$
\frac{d}{d t} \omega_{s}^{(\infty)}(t, x)=\frac{i 2 \pi}{p} \omega_{s / p}^{(\infty)}(t, x)
$$

Из этого уравнения, используя (32), можно получить, что $\forall f \in \mathbf{T}_{p}, \forall t \in \mathbf{R}$

$$
\frac{d}{d t} \Omega_{s, a}^{(\infty)}\left(f_{t}\right)=\Gamma\left(\Omega_{s, a}^{(\infty)}\left(f_{t}\right)\right)
$$

Отсюда в соответствии с теорией дифференциальных уравнений [17] следует формула (38).

Укажем еше одну специфическую особенность случая $m=\infty$. Из формул $(14),(15)$, (28), (30) вытекает, что $\forall x \in \mathbf{Q}_{p}$

$$
\left[\Upsilon_{s}^{(\infty)}\right](x)=\omega_{s}^{(\infty)}\left(\{x\}_{p},[x]_{p}\right)
$$



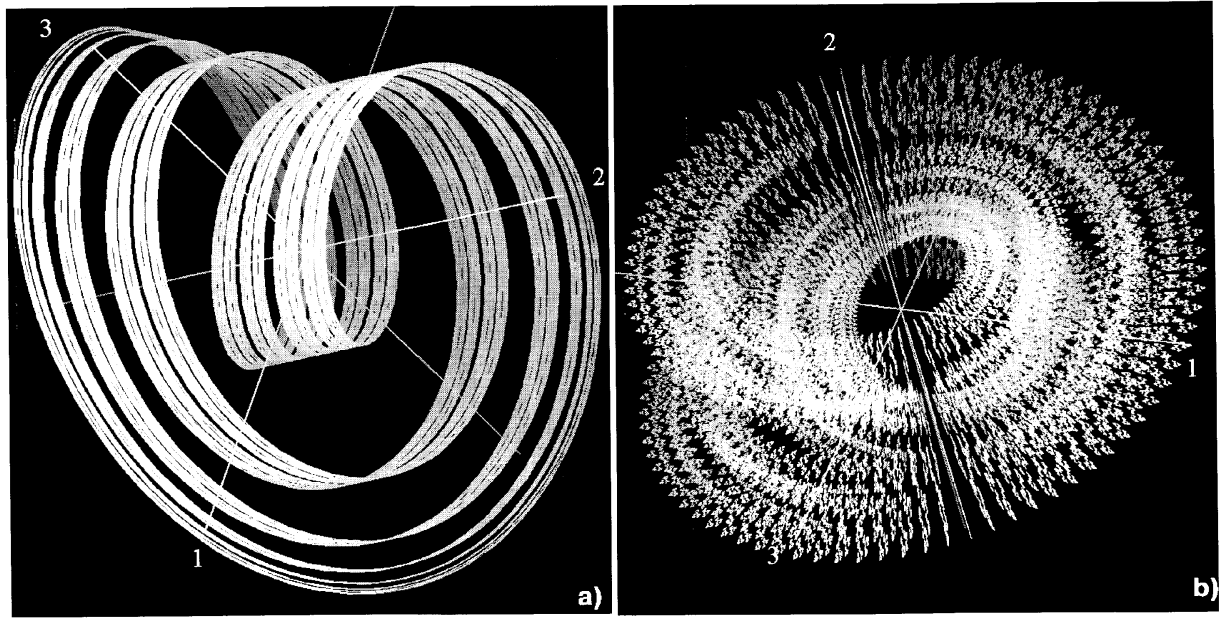

Рис. 2. Вложения в $\mathbf{R}^{3}$ : a) $\mathbf{T}_{2}(m=0)$; b) $\mathbf{T}_{3}$ (слоями) или $3^{-4} \mathbf{Z}_{3}(m=\infty)$.

Отсюда следует упомянутое вьше равенство $\tilde{\Delta}_{s}^{\infty}=\Delta_{s}^{\infty}$. Кроме того, используя теперь теорему 5 и теорему Кирсбрауна [8], можно показать, что если $\Omega_{s, a}^{(\infty)}-L$-изометрия, то найдется $L$-сжатие $J: \mathbf{C} \mapsto \mathbf{R}^{3}$, такое что $J$ отображает изометрично любой кластер $B_{l}^{0}=\Upsilon_{s}^{(\infty)}\left(\left\{x \in \mathbf{Q}_{p}:|x-l|_{p}<1\right\}\right)$ на образ $\{l\}_{p^{-}}$слоя $\Omega_{s, a}^{(\infty)}\left(\left(\{l\}_{p}, \mathbf{Z}_{p}\right)\right)$ и

$$
\Omega_{s, a}^{(\infty)} \circ j=J \circ \Upsilon_{s}^{(\infty)}
$$

\section{5. ПОЯСНЕНИЯ К РИСУНКАМ}

Благодаря хорошей сходимости рядов (6) и (15), а также поскольку даже не очень большие натуральные числа образуют достаточно плотную сетку в $\mathbf{Z}_{p}$, принципиальных трудностей для компьютерных построений множеств $\Upsilon_{s}^{(m)}\left(\mathbf{Z}_{p}\right)$ и $\Omega_{s, a}^{(m)}\left(\mathbf{T}_{p}\right)$ не возникает.

На рис. 1.1 изображено множество Кантора и для наглядности каждой точке сопоставлен отрезок длины, пропорциональной одному из чисел $e_{i}=1,3,5,7,2,6,10,14$, если точка образа имеет вид $\Upsilon_{1 / 3}^{(0)}\left(e_{i} \cdot y^{2}\right)$ для $y \in \mathbf{Z}_{p}$ (см. об этих числах [1]). Множество на рис. 1.10 есть, очевидно, ковер Серпинского. Из рис. 1.9 видно, что границы связанных компонент множества $\mathbf{C} \backslash \Upsilon_{1 / 3}^{(0)}\left(\mathbf{Z}_{6}\right)$ состоят из кривых Коха (заметим, что $p$ не простое, см. сноску 3). И наконец, рис. 1.4 может служить иллюстрацией того, что $\mathbf{Z}_{4}$ гомеоморфно $\mathbf{Z}_{2}$.

На рис. 2а построено вложение $\mathbf{T}_{2}$ в $\mathbf{R}^{3}$ с параметрами $s=1 / 2.2, a=i 2$ и $m=0$, отчетливо видна канторо-подобная структура этого множества. На рис. $2 \mathrm{~b}$ построено вложение $\mathbf{T}_{3}\left(s=s_{0}-0.02 \approx 0.46, a=5 / 2\right.$ и $\left.m=\infty\right)$. Показаны слои со значениями $\xi$, кратными $1 / 81$, поэтому рис. $2 \mathrm{~b}$ можно одновременно рассматривать как образ $\Upsilon_{s}^{(\infty)}\left(3^{-4} \mathbf{Z}_{3}\right)$ при отображении $J$ (см. формулы $\left.(40),(41)\right)$, показанный фактически на рис. 1.12 .

В заключение отметим, что по крайней мере для $m=\infty$ можно аналогичным образом рассматривать и более общие конструкции, такие как $a$-адические числа и соленоиды (см. [11]). При этом, по-видимому, представляет интерес, например, построение 
вложения группы $\mathbf{A} / \mathbf{Q}$ в $\mathbf{R}^{3}$, где $\mathbf{A}$ - кольцо аделей [18].

\section{Список литературы}

[1] В.С. Владимиров, И.В. Волович, Е.И. Зеленов. р-Адический анализ и математическая физика. М.: Наука, 1994.

[2] M. Pitkanen. p-adic Physics? Preprint University of Helsinki, SF-00170, 1994

[3] S. Havlin, N. Weissman. J. Phys. A. 1986. V. 19. P. 1021-1026.

[4] E.I. Zelenov. J. Math. Phys. 1991. V. 32. P. 147-152.

[5] А. Лихтенберг, М. Либерман. Регулярная и стохастическая динамика. М.: Мир, 1984.

[6] A.T. Ogielski, D.L. Stein. Phys. Rev. Let. 1985. V. 55. № 15.

[7] K. Аллен, M. Клуатр. Оптические преобразования фракталов. Фракталы в физике /Ред. Л. Пьетронеро, Э. Тозатти. М.: Мир, 1988.

[8] Г. Федерер. Геометрическая теория меры. М.: Мир, 1981.

[9] П. Биллингслей. Эргодическая теория и информация. М.: Мир, 1969.

[10] Н. Коблии. p-Адические числа, $p$-адический анализ и дзета-функции. М.: Мир, 1982.

[11] Э. Хьюит, К. Росс. Абстрактный гармонический анализ. Т. 1. М.: Наука, 1975.

[12] Джс. Келли. Общая топология. М.: Наука, 1981.

[13] И.М. Гельфанд, М.И. Граев, Л.И. Пятецкий-Шапиро. Обобщенные функции. Вып. 6. М.: Наука, 1966.

[14] Б.В. Шабат. Комплексный анализ. Т. 1. М.: Наука, 1985.

[15] Е. Федер. Фракталы. М.: Мир, 1991.

[16] Джс. Займан. Модели беспорядка. М.: Мир, 1982.

[17] В.И. Арнольд. Обыкновенные дифференциальные уравнения. М.: Наука, 1975.

[18] Д. Мамфорд. Лекции о тета-функциях. М.: Мир, 1988.

Поступила в редакцию 21.V.1996 г.

\section{V. Chistyakov}

\section{FRACTAL GEOMETRY OF IMAGES OF $p$-ADIC NUMBERS AND SOLENOIDS CONTINUOUS IMMERSIONS TO EUCLIDEAN SPACES}

Family of continuous maps of $p$-adic numbers $\mathbf{Q}_{p}$ and solenoids $\mathbf{T}_{p}$ to the complex plane $\mathbf{C}$ and to the $\mathbf{R}^{3}$, respectively, are obtained in an explicit form. Maps for which the Cantor set and the Serpinsky triangle are unitary ball images to $\mathbf{Q}_{2}$ and $\mathbf{Q}_{3}$, respectively, belong to such families. The subset of immersions for each of that families is found. For these immersions Hausdorff dimensions of images are calculated and it is shown that fractal measure of $\mathbf{Q}_{p}$ image coincides with the Haar measure in $\mathbf{Q}_{p}$. It is shown, that the image of the $p$-adic solenoid is invariant set with fractal dimension of a some dynamic system. Computer pictures of some fractal images are presented. 